Internet Engineering Task Force (IETF)

Request for Comments: 5942

Updates: 4861

Category: Standards Track

ISSN : $2070-1721$
H. Singh

W. Beebee

Cisco Systems, Inc.

E. Nordmark

Oracle, Inc.

July 2010

IPv6 Subnet Model: The Relationship between Links and Subnet Prefixes

\title{
Abstract
}

IPv6 specifies a model of a subnet that is different than the IPv4 subnet model. The subtlety of the differences has resulted in incorrect implementations that do not interoperate. This document spells out the most important difference: that an IPv6 address isn't automatically associated with an IPv6 on-link prefix. This document also updates (partially due to security concerns caused by incorrect implementations) a part of the definition of "on-link" from RFC 4861.

Status of This Memo

This is an Internet Standards Track document.

This document is a product of the Internet Engineering Task Force (IETF). It represents the consensus of the IETF community. It has received public review and has been approved for publication by the Internet Engineering Steering Group (IESG). Further information on Internet Standards is available in Section 2 of RFC 5741.

Information about the current status of this document, any errata, and how to provide feedback on it may be obtained at

http://www.rfc-editor.org/info/rfc5942.

Copyright Notice

Copyright (c) 2010 IETF Trust and the persons identified as the document authors. All rights reserved.

This document is subject to BCP 78 and the IETF Trust's Legal Provisions Relating to IETF Documents

(http://trustee.ietf.org/license-info) in effect on the date of publication of this document. Please review these documents carefully, as they describe your rights and restrictions with respect to this document. Code Components extracted from this document must include Simplified BSD License text as described in section $4 . e$ of the Trust Legal Provisions and are provided without warranty as described in the Simplified BSD License. 
This document may contain material from IETF Documents or IETF Contributions published or made publicly available before November 10, 2008. The person(s) controlling the copyright in some of this material may not have granted the IETF Trust the right to allow modifications of such material outside the IETF Standards Process. Without obtaining an adequate license from the person(s) controlling the copyright in such materials, this document may not be modified outside the IETF standards Process, and derivative works of it may not be created outside the IETF Standards Process, except to format it for publication as an RFC or to translate it into languages other than English.

Table of Contents

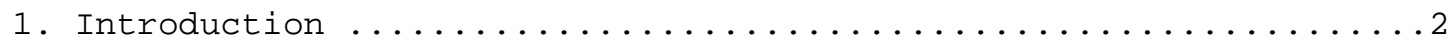



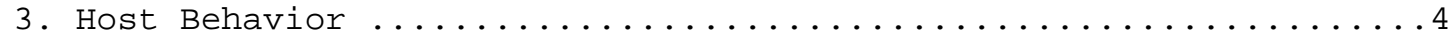

4. Host Rules ...............................

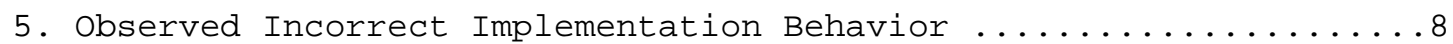



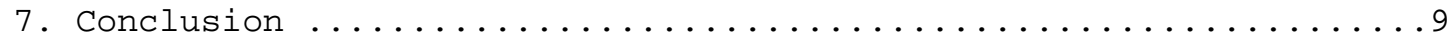

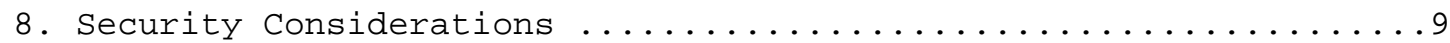

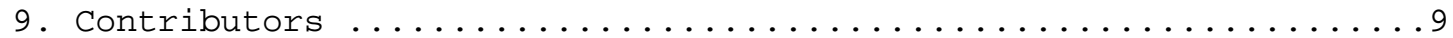

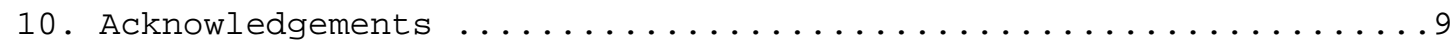

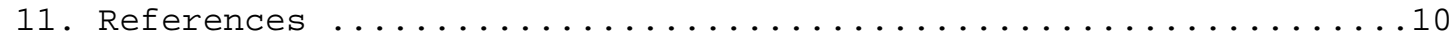

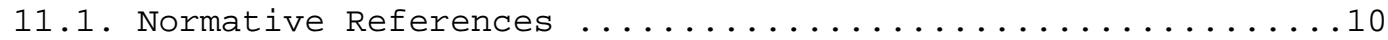

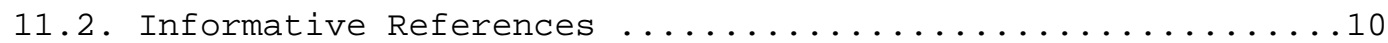

1. Introduction

IPv4 implementations typically associate a netmask with an address when an IPv4 address is assigned to an interface. That netmask together with the IPv4 address designates an on-link prefix. Nodes consider addresses covered by an on-link prefix to be directly attached to the same link as the sending node, i.e., they send traffic for such addresses directly rather than to a router. See Section 3.3.1 of [RFC1122]. Prior to the development of subnetting [RFC0950] and Classless Inter-Domain Routing (CIDR) [RFC4632], an address's netmask could be derived directly from the address simply by determining whether it was a Class A, B, or C address. Today, assigning an address to an interface also requires specifying a netmask to use. In the absence of specifying a specific netmask when assigning an address, some implementations would fall back to deriving the netmask from the class of the address.

The behavior of IPv6 as specified in Neighbor Discovery (ND) [RFC4861] is quite different. The on-link determination is separate from the address assignment. A host can have IPv6 addresses without 
any related on-link prefixes or can have on-link prefixes that are not related to any IPV6 addresses that are assigned to the host. Any assigned address on an interface should initially be considered as having no internal structure as shown in [RFC4291].

In IPv6, by default, a host treats only the link-local prefix as on-link.

The reception of a Prefix Information Option (PIO) with the L-bit set [RFC4861] and a non-zero valid lifetime creates (or updates) an entry in the Prefix List. All prefixes on a host's Prefix List (i.e., those prefixes that have not yet timed out) are considered to be on-link by that host.

The on-link definition in the Terminology section of [RFC4861], as modified by this document, defines the complete list of cases in which a host considers an address to be on-link. Individual address entries can be expired by the Neighbor Unreachability Detection mechanism.

IPv6 packets sent using the Conceptual Sending Algorithm as described in [RFC4861] only trigger address resolution for IPv6 addresses that the sender considers to be on-link. Packets to any other address are sent to a default router. If there is no default router, then the node should send an ICMPv6 Destination Unreachable indication as specified in [RFC4861] -- more details are provided in the "Host Behavior" and "Host Rules" sections of this document. (Note that [RFC4861] changed the behavior when the Default Router List is empty.

In the old version of Neighbor Discovery [RFC2461], if the Default Router List is empty, rather than sending the ICMPv6 Destination Unreachable indication, the [RFC2461] node assumed that the destination was on-link.) Note that ND is scoped to a single link. All Neighbor Solicitation (NS) responses are assumed to be sent out the same interface on which the corresponding query was received without using the Conceptual Sending Algorithm.

Failure of host implementations to correctly implement the IPv6 subnet model can result in lack of IPv6 connectivity. See the "Observed Incorrect Implementation Behavior" section for details.

This document deprecates the last two bullets from the definition of "on-link" in [RFC4861] to address security concerns arising from particular ND implementations.

Host behavior is clarified in the "Host Behavior" and "Host Rules" sections. 


\section{Requirements Language}

The key words "MUST", "MUST NOT", "REQUIRED", "SHALL", "SHALL NOT", "SHOULD", "SHOULD NOT", "RECOMMENDED", "MAY", and "OPTIONAL" in this document are to be interpreted as described in RFC 2119 [RFC2119].

\section{Host Behavior}

1. The original Neighbor Discovery (ND) specification [RFC4861] was unclear in its usage of the term "on-link" in a few places. In IPv6, an address is on-link (with respect to a specific link), if the address has been assigned to an interface attached to that link. Any node attached to the link can send a datagram directly to an on-link address without forwarding the datagram through a router. However, in order for a node to know that a destination is on-link, it must obtain configuration information to that effect. In IPv6, there are two main ways of maintaining information about on-link destinations. First, a host maintains a Prefix List that identifies ranges of addresses that are to be considered on-link. Second, Redirects can identify individual destinations that are on-link; such Redirects update the Destination Cache.

The Prefix List is populated via the following means:

* Receipt of a valid Router Advertisement (RA) that specifies a prefix with the L-bit set. Such a prefix is considered on-link for a period specified in the Valid Lifetime and is added to the Prefix List. (The link-local prefix is effectively considered a permanent entry on the Prefix List.)

* Indication of an on-link prefix (which may be a /128) via manual configuration, or some other yet-to-be-specified configuration mechanism.

A Redirect can also signal whether an address is on-link. If a host originates a packet, but the first-hop router routes the received packet back out onto the same link, the router also sends the host a Redirect. If the Target and Destination Address of the Redirect are the same, the Target Address is to be treated as on-link as specified in section 8 of [RFC4861]. That is, the host updates its Destination Cache (but not its Prefix List -though the impact is similar).

2. It should be noted that ND does not have a way to indicate a destination is "off-link". Rather, a destination is assumed to be off-link, unless there is explicit information indicating that it is on-link. Such information may later expire or be changed, 
in which case a destination may revert back to being considered off-link, but that is different than there being an explicit mechanism for signaling that a destination is off-link. Redirect messages do not contain sufficient information to signal that an address is off-link. Instead, Redirect messages indicate a preferred next hop that is a more appropriate choice to use than the originator of the Redirect.

3. IPv6 also defines the term "neighbor" to refer to nodes attached to the same link and that can send packets directly to each other. Received ND packets that pass the required validation tests can only come from a neighbor attached to the link on which the ND packet was received. Unfortunately, [RFC4861] is imprecise in its definition of "on-link" and states that a node considers an address to be on-link if:

* a Neighbor Advertisement (NA) message is received for the (target) address, or

* any Neighbor Discovery message is received from the address.

Neither of these tests are acceptable definitions for an address to be considered as on-link as defined above, and this document deprecates and removes both of them from the formal definition of "on-link". Neither of these tests should be used as justification for modifying the Prefix List or Destination Cache for an address.

The conceptual sending algorithm of [RFC4861] defines a Prefix List, Destination Cache, and Default Router List. The combination of Prefix List, Destination Cache, and Default Router List form what many implementations consider to be the IP data forwarding table for a host. Note that the Neighbor Cache is a separate data structure referenced by the Destination Cache, but entries in the Neighbor Cache are not necessarily in the Destination Cache. It is quite possible (and intentional) that entries be added to the Neighbor Cache for addresses that would not be considered on-link as defined above. For example, upon receipt of a valid NS, Section 7.2.3 of [RFC4861] states:

If an entry does not already exist, the node SHOULD create a new one and set its reachability state to STALE as specified in section 7.3.3. If an entry already exists, and the cached link-layer address differs from the one in the received Source Link-Layer option, the cached address should be replaced by the received address, and the entry's reachability state MUST be set to STALE. 
The intention of the above feature is to add an address to the Neighbor Cache, even though it might not be considered on-link per the Prefix List. The benefit of such a step is to have the receiver populate the Neighbor Cache with an address it will almost certainly be sending packets to shortly, thus avoiding the need for an additional round of ND to perform address resolution. But because there is no validation of the address being added to the Neighbor Cache, an intruder could spoof the address and cause a receiver to add an address for a remote site to its Neighbor Cache. This vulnerability is a specific instance of the broad set of attacks that are possible by an on-link neighbor [RFC3756]. This causes no problems in practice, so long as the entry only exists in the Neighbor Cache and the address is not considered to be on-link by the IP forwarding code (i.e., the address is not added to the Prefix List and is not marked as on-link in the Destination Cache).

4. After the update to the on-link definition in [RFC4861], certain text from Section 7.2.3 of [RFC4861] may appear, upon a cursory examination, to be inconsistent with the updated definition of "on-link" because the text does not ensure that the source address is already deemed on-link through other methods:

If the Source Address is not the unspecified address and, on link layers that have addresses, the solicitation includes a Source Link-Layer Address option, then the recipient SHOULD create or update the Neighbor Cache entry for the IP source Address of the solicitation.

Similarly, the following text from Section 6.2.6 of [RFC4861] may also seem inconsistent:

If there is no existing Neighbor Cache entry for the solicitation's sender, the router creates one, installs the link-layer address and sets its reachability state to STALE as specified in Section 7.3.3.

However, the text in the aforementioned sections of [RFC4861], upon closer inspection, is actually consistent with the deprecation of the last two bullets of the on-link definition because there are two different ways in which on-link determination can affect the state of ND: through updating the Prefix List or updating the Destination Cache. Through deprecating the last two bullets of the on-link definition, the Prefix List is explicitly not to be changed when a node receives an NS, NA, or Router Solicitation (RS). The Neighbor Cache can still be updated through receipt of an NS, NA, or RS. 
5. [RFC4861] is written from the perspective of a host with a single interface on which Neighbor Discovery is run. All ND traffic (whether sent or received) traverses the single interface. On hosts with multiple interfaces, care must be taken to ensure that the scope of ND processing from one link stays local to that link. That is, when responding to an NS, the NA would be sent out on the same link on which it was received. Likewise, a host would not respond to a received NS for an address only assigned to an interface on a different link. Although implementations may choose to implement Neighbor Discovery using a single data structure that merges the Neighbor Caches of all interfaces, an implementation's behavior must be consistent with the above model .

4. Host Rules

A correctly implemented IPv6 host MUST adhere to the following rules:

1. The assignment of an IPv6 address -- whether through IPV6 stateless address autoconfiguration [RFC4862], DHCPV6 [RFC3315], or manual configuration -- MUST NOT implicitly cause a prefix derived from that address to be treated as on-link and added to the Prefix List. A host considers a prefix to be on-link only through explicit means, such as those specified in the on-link definition in the Terminology section of [RFC4861] (as modified by this document) or via manual configuration. Note that the requirement for manually configured addresses is not explicitly mentioned in [RFC4861].

2. In the absence of other sources of on-link information, including Redirects, if the RA advertises a prefix with the on-link (L) bit set and later the Valid Lifetime expires, the host MUST then consider addresses of the prefix to be off-link, as specified by the PIO paragraph of Section 6.3.4 of [RFC4861].

3. In the absence of other sources of on-link information, including Redirects, if the RA advertises a prefix with the on-link (L) bit set and later the Valid Lifetime expires, the host MUST then update its Prefix List with respect to the entry. In most cases, this will result in the addresses covered by the prefix defaulting back to being considered off-link, as specified by the PIO paragraph of Section 6.3.4 of [RFC4861]. However, there are cases where an address could be covered by multiple entries in the Prefix List, where expiration of one prefix would result in destinations then being covered by a different entry. 
4. Implementations compliant with [RFC4861] MUST adhere to the following rules. If the Default Router List is empty and there is no other source of on-link information about any address or prefix:

a. The host MUST NOT assume that all destinations are on-link.

b. The host MUST NOT perform address resolution for non-linklocal addresses.

c. Since the host cannot assume the destination is on-link, and off-link traffic cannot be sent to a default router (since the Default Router List is empty), address resolution cannot be performed. This case is specified in the last paragraph of Section 4 of [RFC4943]: when there is no route to the destination, the host should send an ICMPv6 Destination Unreachable indication (for example, a locally delivered error message) as specified in the Terminology section of [RFC4861].

On-link information concerning particular addresses and prefixes can make those specific addresses and prefixes on-link, but does not change the default behavior mentioned above for addresses and prefixes not specified. [RFC4943] provides justification for these rules.

5. Observed Incorrect Implementation Behavior

One incorrect implementation behavior illustrates the severe consequences when the IPv6 subnet model is not understood by the implementers of several popular host operating systems. In an access concentrator network ([RFC4388]), a host receives a Router Advertisement message with no on-link prefix advertised. An address could be acquired through the DHCPv6 identity association for nontemporary addresses (IA_NA) option from [RFC3315] (which does not include a prefix length), or through manual configuration (if no prefix length is specified). The host incorrectly assumes an invented prefix is on-link. This invented prefix typically is a /64 that was written by the developer of the operating system network module API to any IPv6 application as a "default" prefix length when a length isn't specified. This may cause the API to seem to work in the case of a network interface initiating stateless address autoconfiguration (SLAAC); however, it can cause connectivity problems in Non-Broadcast Multi-Access (NBMA) networks. Having incorrectly assumed an invented prefix, the host performs address resolution when the host should send all non-link-local traffic to a 
default router. Neither the router nor any other host will respond to the address resolution, preventing this host from sending IPv6 traffic.

6. Updates to RFC 4861

This document deprecates the following two bullets from the on-link definition in Section 2.1 of [RFC4861]:

- a Neighbor Advertisement message is received for the (target) address, or

- any Neighbor Discovery message is received from the address.

7. Conclusion

This document clarifies and summarizes the relationship between links and subnet prefixes described in [RFC4861]. Configuration of an IPv6 address does not imply the existence of corresponding on-link prefixes. One should also look at API considerations for prefix length as described in the last paragraph of Section 4.2 of [RFC4903]. This document also updates the definition of "on-link" from [RFC4861] by deprecating the last two bullets.

8. Security Considerations

This document addresses a security concern present in [RFC4861]. As a result, the last two bullets of the on-link definition in [RFC4861] have been deprecated. US-CERT Vulnerability Note VU\#472363 lists the implementations affected.

9. Contributors

Thomas Narten contributed significant text and provided substantial guidance to the production of this document.

\section{Acknowledgements}

Thanks (in alphabetical order) to Adeel Ahmed, Jari Arkko, Ralph Droms, Alun Evans, Dave Forster, Prashanth Krishnamurthy, Suresh Krishnan, Josh Littlefield, Bert Manfredi, David Miles, Madhu Sudan, Jinmei Tatuya, Dave Thaler, Bernie Volz, and Vlad Yasevich for their consistent input, ideas, and review during the production of this document. The security problem related to an NS message that provides one reason for invalidating a part of the on-link definition was found by David Miles. Jinmei Tatuya found the security problem to also exist with an RS message. 
11. References

11.1. Normative References

[RFC2119] Bradner, S., "Key words for use in RFCs to Indicate Requirement Levels", BCP 14, RFC 2119, March 1997.

[RFC4861] Narten, T., Nordmark, E., Simpson, W., and H. Soliman, "Neighbor Discovery for IP version 6 (IPv6)", RFC 4861, September 2007 .

11.2. Informative References

[RFC0950] Mogul, J. and J. Postel, "Internet Standard Subnetting Procedure", STD 5, RFC 950, August 1985.

[RFC1122] Braden, R., "Requirements for Internet Hosts Communication Layers", STD 3, RFC 1122, October 1989.

[RFC2461] Narten, T., Nordmark, E., and W. Simpson, "Neighbor Discovery for IP Version 6 (IPv6)", RFC 2461, December 1998 .

[RFC3315] Droms, R., Bound, J., Volz, B., Lemon, T., Perkins, C., and M. Carney, "Dynamic Host Configuration Protocol for IPv6 (DHCPv6)", RFC 3315, July 2003.

[RFC3756] Nikander, P., Kempf, J., and E. Nordmark, "IPv6 Neighbor Discovery (ND) Trust Models and Threats", RFC 3756, May 2004 .

[RFC4291] Hinden, R. and S. Deering, "IP Version 6 Addressing Architecture", RFC 4291, February 2006.

[RFC4388] Woundy, R. and K. Kinnear, "Dynamic Host Configuration Protocol (DHCP) Leasequery", RFC 4388, February 2006.

[RFC4632] Fuller, V. and T. Li, "Classless Inter-domain Routing (CIDR): The Internet Address Assignment and Aggregation Plan", BCP 122, RFC 4632, August 2006.

[RFC4862] Thomson, S., Narten, T., and T. Jinmei, "IPv6 Stateless Address Autoconfiguration", RFC 4862, september 2007.

[RFC4903] Thaler, D., "Multi-Link Subnet Issues", RFC 4903, June 2007 . 
[RFC4943] Roy, S., Durand, A., and J. Paugh, "IPv6 Neighbor Discovery On-Link Assumption Considered Harmful", RFC 4943, September 2007.

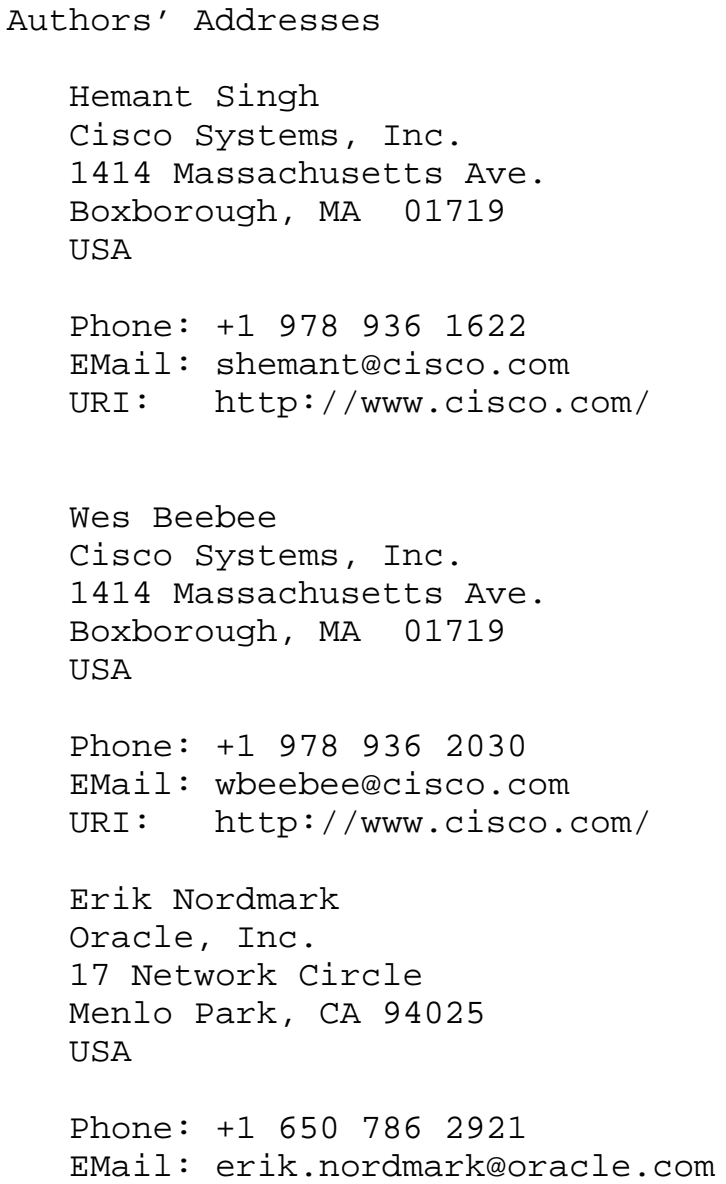

